\title{
CORRIGENDUM
}

\section{Collisional alpha transport in a weakly non-quasisymmetric stellarator magnetic field - CORRIGENDUM}

\author{
Peter J. Catto \\ doi:10.1017/S0022377819000321, Published by Cambridge University Press, \\ 2 May 2019
}

The symbol on the left side of (3.13) should be the same as defined in (2.12), namely, $\Theta(\alpha, \eta)$.

The two $q N-M$ factors in (2.21) should be replaced with $N-q^{-1} M$. Then, above (2.27) the statement should read 'the radial variation of $N-q^{-1} M$ is negligible if $1-q N / M \gg R q^{-1} \partial q / \partial r^{\prime}$.

In addition, for the superbanana plateau regime (sbp) the evaluation of (3.13) needs to be slightly different than the $\sqrt{v}$ regime procedure presented in (3.14) to (3.16). For the sbp regime the boundary layer is at $\kappa_{0}^{2} \simeq 0.83$, rather than the trapped-passing boundary. Therefore, $\eta_{t}$ should be replaced by $\eta_{0} \equiv 2 \sin ^{-1} \kappa_{0} \simeq 2.3$ in (3.13), (3.14) and (3.15) for the sbp case. As a result, the $\cos [(q n-m) \pi /(q N-M)]$ term in (3.16), (3.18), (7.8), (7.13), (7.14), (7.16) and (7.17) should be replaced by $\cos \left[(q n-m) \eta_{0} /(q N-M)\right]$. For the same reason, $\cos (q n \pi)$ must be replaced by $\cos \left(q n \eta_{0}\right)$ in (7.18) and (7.19).

\section{Acknowledgements}

Work supported by the US Department of Energy grant DE-FG02-91ER-54109.

\section{REFERENCE}

CATTO, P. J. 2019 Collisional alpha transport in a weakly non-quasisymmetric stellarator magnetic field. J. Plasma Phys 85, 905850213. 